\title{
Dispersion refinements in the range of absorption edges
}

\author{
Florian Meurer ${ }^{1}$, Florian Kleemiss ${ }^{1}$, Oleg V. Dolomanov², Vedran Vuković ${ }^{3}$, Horst Puschmann ${ }^{2}$, \\ Norbert Peyerimhoff ${ }^{4}$, Christoph Hennig ${ }^{5}$, Michael Bodensteiner ${ }^{1}$ \\ ${ }^{1}$ Universität Regensburg, Fakultät für Chemie und Pharmazie, Universitätsstr. 31, 93053 Regensburg, Germany, \\ ${ }^{2}$ OlexSys Ltd, Chemistry Department, Durham University, DH1 3LE, UK, \\ ${ }^{3}$ Université de Lorraine, CNRS, CRM2, 54000 Nancy, France, \\ ${ }^{4}$ Department of Mathematical Sciences, Durham University, DHI 3LE, UK, \\ ${ }^{5}$ Rossendorf Beamline (BM20-CRG), European Synchrotron Radiation Facility, 71, Avenue des Martyrs, 38043 Grenoble, France \\ michael.bodensteiner@ur.de
}

Most crystallographers avoid performing experiments in the area around an absorption edge of an involved heavy element. Although standard refinement procedures account for dispersion effects, the structure models tend to become unreliable in this range of energies. This originates from the fact that tabulated dispersion values $f^{\prime}$ and $f$ " are used. Tables from different sources differ significantly from one another and do not take the chemical environment of atoms in a crystal structure into account at all. In contrast, XAFS spectroscopists are particularly interested in the energy range around absorption edges and derive valuable information from it.

Experiments in our home lab at four different wavelengths $(\mathrm{Cu} \mathrm{K} \alpha, \mathrm{Cu} K \beta$, Mo $\mathrm{K} \alpha, \mathrm{Ag} \mathrm{K} \alpha)$ and moreover at the European Synchrotron Radiation Facility were carried out to investigate the influence of dispersion in structure models. The presentation reports on the results of an inclusion of dispersion refinement into crystal structure determinations. We observe a good correlation between the absorption spectrum of a given element and the refined dispersion values. Furthermore, the structure model remains unchanged before, after and even at the absorption edge.
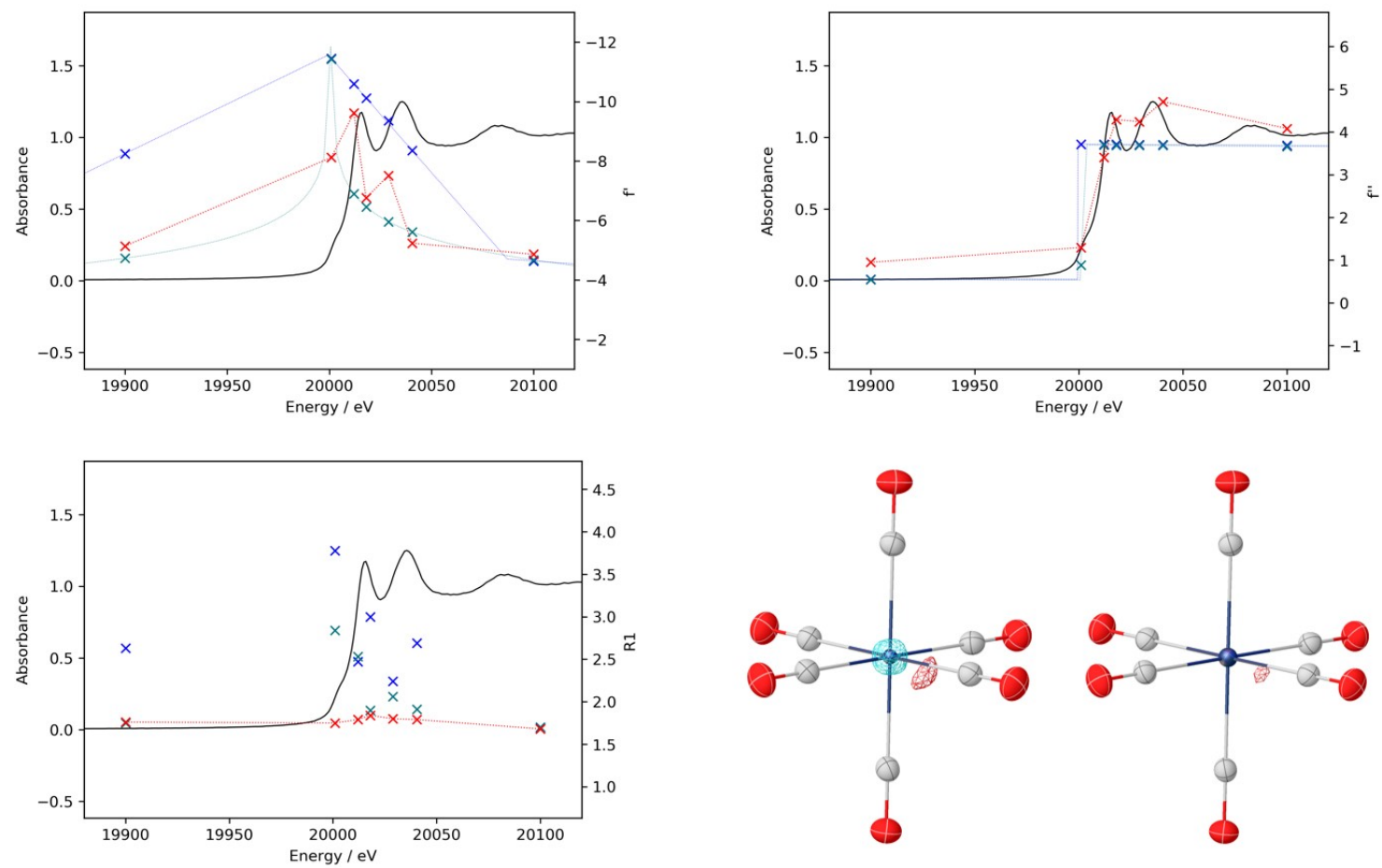

Figure 1. XAFS spectrum (black line) together with tabulated (blue: Henke[1], green: Sasaki[2]) and refined (red) $f^{\prime}, f^{\prime \prime}$ and resulting $R 1$ values. Comparison of the final structure model with Henke[1] (left) and refined dispersion values (right).

[1] Henke, B. L., Gullikson, E. M., Davis, J. C. (1993). At. Data Nucl. Data Tables, 54, 181.

[2] Sasaki, S. (1989). KEK Report, 88, 1.

Keywords: Dispersion, Absorption Edge, Synchrotron 